\title{
MATERIALS SOLIDIFICATION PHYSICS IN SPACE
}

$\gg$ DOI 10.1051/epn:2008502

\author{
L. Ratke ${ }^{1}$, Ch.-A. Gandin ${ }^{2}$, R.H. Mathiesen ${ }^{3}$, M. Rappaz ${ }^{4}$ and S. Rex ${ }^{5}$, \\ ${ }^{1}$ Institut für Materialphysik im Weltraum, DLR Köln, Germany, ${ }^{2}$ Centre de Mise en Forme des Matériaux, ENSMP Sophia Antipolis, France, \\ ${ }^{3}$ Dept. of Physics, NTNU Trondheim, Norway, ${ }^{4}$ Institut des Matériaux, EPFL Lausanne, Switzerland, ${ }^{5}$ ACCESS e.V., Aachen, Germany
}

○ ne of the major unresolved problems in solidification physics and its industrial counterpart, the foundry industry, reads simply: the effect of fluid flow on the microstructure of cast products. As "cast" is just another word for "forced flow" and casting of alloys is an art and engineering technology of a few thousand years old, it might appear peculiar that the interplay between an advancing solidification front and fluid flow in the molten alloy still remains to be understood.

Solidification physics in general deals with the evolution of microstructures during the solid-liquid phase transformation. The term microstructure here relates to crystalline patterns developing from a complex interplay between heat and mass transport in the melt and the solid, where the solid-liquid interface morphology is defined in the process. In castings, the solidification process always starts with the nucleation of crystals in a region of the melt that is below its equilibrium temperature. This usually occurs at the surface of the mould. As growth of these nuclei is unstable, dendrites grow as trees with primary trunks and side branches parallel to fairly well-defined crystallographic directions. Their selective growth leads to dendritic patterns which are called columnar, since they look like columns or pillars growing parallel to the thermal gradient. The growth of these columnar dendrites can be arrested by the nucleation and growth of dendritic grains forming in the bulk liquid ahead of the columnar dendrite tips. These grains that exhibit dendritic arms growing in different radial directions are called equiaxed and the transition is the Columnar-toEquiaxed Transition (CET). In practice, this transition is either avoided or triggered to proceed as quickly as possible in order to obtain samples with homogeneous microstructure.
The region where dendrites, whether columnar or equiaxed, develop and are still in contact with the liquid is called the mushy zone. An illustration of the CET and the dendritic microstructure is given in Fig. 1.

\section{Convection}

Despite several models for the CET, the influence of convection is still an open question in solidification physics. Numerous theoretical and experimental papers have been published and a few important mechanisms seem to be responsible [1]. The European programme "Columnar to Equiaxed Transition in SOLidification processing (CETSOL)" aims to investigate this transition and to identify the underlying mechanisms. Microgravity processing on sounding rockets, essential to avoid buoyancy induced fluid-flows, reveals that CET in directionally solidified $\mathrm{Al}-7 \mathrm{wt} \% \mathrm{Si}$ alloys occurred earlier than in the corresponding ground environment.

The growth of dendrites in metallic alloys depends on many parameters, such as alloy composition, cooling rate, interface velocity, temperature and solute gradients at the solid-liquid interface, thermophysical parameters such as concentration and temperature - dependent diffusion coefficients, interfacial energy, kinetics of atoms attachment at the solid-liquid interface etc. [2, 3].

The development of physically-based models of the evolution of microstructures is the core of solidification physics. This is especially the case with respect to engineering alloys used, e.g., in crankcases or cylinder heads, turbine blades and cooking pans or as an ingot for sheet metal production. The microstructure as retrieved from the mould is decisive for many properties, including mechanical, electrical, thermal, corrosion resistance,

V FIG. 1: Columnar-to-equiaxed transition (CET) in Al-7wt\%Si alloy solidification carried-out in space. The structure develops from left to right. Starting from a fine equiaxed structure in the stabilized zone, selective growth leads to columnar patterns which end in equiaxed grains. The transition from columnar-to-equiaxed structure is of utmost importance for the foundry industry.

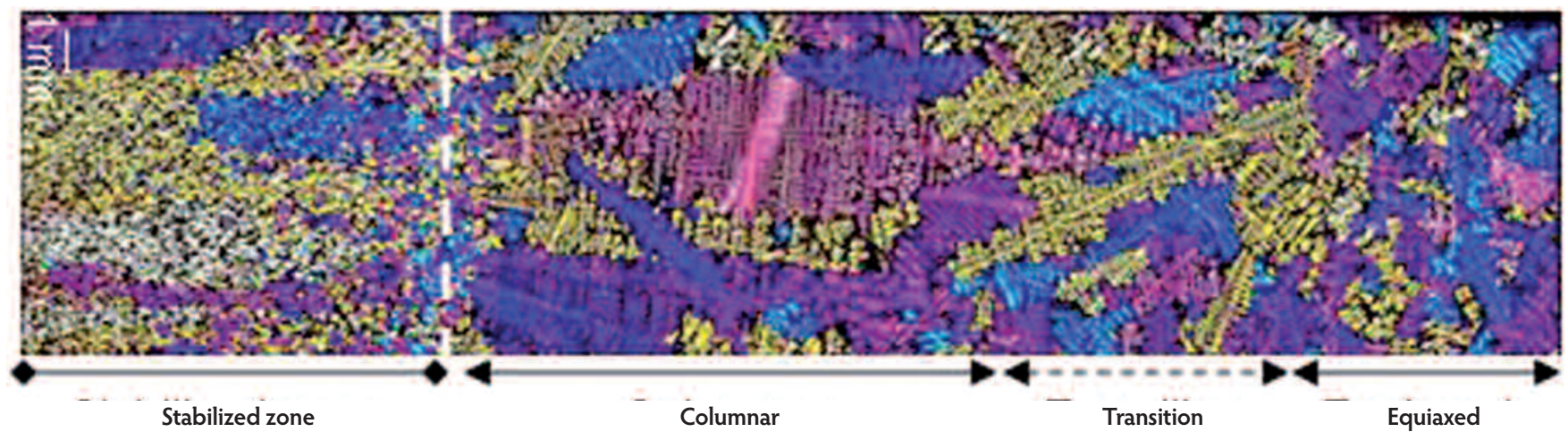


friction and wear, formability and welding properties. Since approximately five decades, the classical foundry practice gradually evolved into solidification physics. Numerous papers and books describe essentials of microstructure evolution under so-called diffusive transport conditions for heat and mass.

Important theoretical milestones are in this respect the discovery of the Mullins-Sekerka instability, marginal stability and microscopic solvability theories providing a basic understanding of dendrite formation. A critical test of these theories was performed with microgravity experiments by Glicksman and coworkers in which established flow-free conditions of heat transfer [4-6] allowed a careful validation of theoretical models.

The same approach is presently under development for modelling of solidification processes with microstructural features. Computer simulations have enabled one to model complex processes better than ever before by combining momentum, heat and mass transport equations for multiple phases. However, the predictive power of such approaches is dependent on benchmark experimental validation.

\section{Forest-like}

The main difficulty lies in the flow interactions with the mushy zone in a way similar to winds or storm interacting with a forest. Does the strong wind above the tip of the trees permeate to the forest soil? Or is it damped considerably before it reaches the ground? This depends essentially on the permeability of the mush. There is no reliable measurement of mush permeability or validated theories for it. The problem is old, and numerous attempts have already been made to solve at least a few issues. Papers have been published investigating the effect of flow on the growth of primary dendrite trunks, the evolution of side branches, the entrainment of solute in the mush [7], the effects of artificial flow induced by rotating or alternating magnetic fields on cast microstructures [8]. However, the understanding of fluid flow on the liquid-to-solid transformation is rather in its infancy and tremendous progress is expected from comparison of simulations with results provided by space experiments.

Therefore, European teams have defined experimental programs under free-fall conditions using short time experiments in parabolic aircraft flights (20 seconds low gravity), sounding rockets (3-12 minutes) and of course the Materials Science Laboratory, MSL, on board the International Space Station, ISS. Here not only diffusive conditions of heat and mass transport can be established but also controlled fluid flow by means of rotating magnetic field devices. A few examples of on-going space projects and first results will illustrate the benefits of experimenting under reduced gravity conditions.
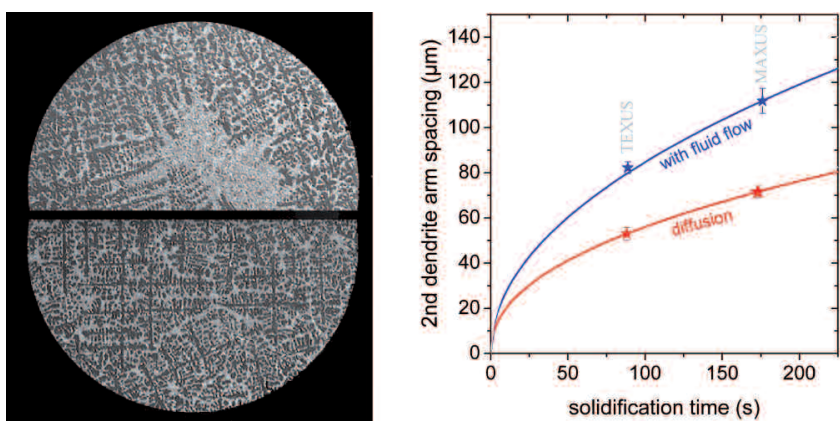

$\Delta$ FIG. 2: Solidification of Al 6wt\%Si alloys in microgravity.

Without fluid flow, a dendritic network develops that is well distributed across the sample (bottom left picture). Fluid flows generated by applying a rotating magnetic field in space result in an enrichment of solute towards the centre of the specimen (top left picture) and also in a different kinetic of the evolution of the dendritic network in the periphery. The right panel depicts the microstructure features of samples processed in pairs in space, one with magnetic convection (curve "with fluid flow") and one without (curve "diffusion"). Experiments were performed on two consecutive missions (Texus and Maxus) providing for the evolution with time in both the flow and no-flow cases. These results from space experiments are coherent with those from laboratory experiments on ground, which are always influenced by a slight natural convection.

\section{New kinetic laws}

The European team of the MICAST project focuses on a systematic analysis of flow effects on dendritic microstructures in Al-base cast alloys starting from academic binary to industrial multi-component systems. Besides extensive laboratory research, experiments on sounding rockets were performed; further experiments will be performed in the MSL. The mush interaction with defined artificial fluid flow induced by rotating magnetic fields was analysed in sounding rocket experiments in the last years. The result shows that new kinetic laws have to be developed to describe the dendrite side branch spacing. Figure 2 shows a result together with a micrograph illustrating the effect of fluid flow: compositional inhomogeneities by segregation of solute on the scale of the cast object. Careful analysis of such microstructures gives the graph to the right. Under purely diffusive transport conditions, the spacing varies with the cubic root of the solidification time. Analytical, simplified models are available for this and implemented in all modern casting software tools. Fluid flow of a few $\mathrm{mm} / \mathrm{s}$ above the mush induces instead a square root-like behaviour, for which first simplified models are just being developed.

Using again the analogy of a dendritic mush with a forest one now might ask, does the interaction of the forest with the wind generate branches, fragments that are taken away with the wind? Such a possible mechanism of fragmentation leading to 
- equiaxed crystals transported into the melt above the mush is beautifully illustrated by in situ synchrotron X-ray radiography during directional solidification of thin films of an $\mathrm{Al}-\mathrm{Cu}$ alloy, showing how dendrite branches melt off and buoy upwards against gravity due to their lower density [9]. If a sufficient number of fragments accumulate above the mushy zone, columnar solidification is no longer possible (movies are available at http://tinyurl.com/3zytrk). These and similar investigations have led to the definition of a European project, XRMON, that aims to develop an X-ray radiographic facility for sounding rockets, thus permitting the study of various solidification phenomena without any disturbances by convection.

\section{New microstructures in bronze}

The casting of steel, done every day on a million-ton basis, is characterised by a so-called peritectic reaction-transformation. This means that below a certain temperature, a new phase nucleates and grows at the periphery of the already formed dendrites under normal solidification conditions. Although practised for thousands of years (this reaction occurs also in bronze and brass castings) the formation of peritectic microstructures has received comparatively little attention. For example, at very low growth rate, novel microstructures were predicted as early as the sixties but were not observed until recently. In particular, under certain conditions, it was anticipated that the primary and the peritectic phases could grow side by side with a planar front instead of one forming around dendrites of the other. This growth morphology, typical of eutectic alloys, is called coupled growth.

The European project METCOMP is focused on the directional solidification at very low speed of peritectic bronze alloys [10]. During this project, coupled growth in $\mathrm{Cu}-\mathrm{Sn}$ alloys was observed for the first time as shown in Fig. 3. This transverse cross section taken at two regions of a single specimen exhibits various coupled growth morphologies, with the (primary) $\alpha$ phase in light yellow-brown and the (peritectic) $\beta$-phase in grey. On the left, the $\alpha$-phase is continuous and the $\beta$-phase is either in the form of lamellae or fibres. On the right, this is the opposite, the $\beta$-phase is continuous and the $\alpha$-phase is in the form of fibres or short lamellae. These morphologies, which are quite similar to recent 3D multi-phase field computations performed by M. Plapp for various eutectic alloys [11], clearly show that the tin composition was not uniform in this specimen. Indeed, both the $\alpha$ - and $\beta$-phases reject the lighter $S n$ element ahead of the $\alpha-\beta$ coupled front, thus inducing solutal natural convection and variations in the local composition. Although convection was reduced in this case by the use of fairly small specimen sizes, it is essential to perform similar directional solidification experiments in a microgravity environment, in which the convection and the associated macrosegregation are decreased substantially.

The upcoming possibilities to experiment in the Materials Science Laboratory onboard the ISS gives the chance to perform systematic experiments in reduced gravity to clarify many aspects of flow effects on pattern formation in solidification.

\section{References}

[1] J.A. Spittle, Int. Mat. Rev. 51 (2006), 247

[2] W. Kurz, D.J. Fisher, Fundamentals of Solidification, Trans. Tech. Publ., Ackermannsdorf, 1989

[3] U. Hecht et al., Mat.Sci.Eng.R 46 (2004), 1

[4] M.E. Glicksman et al., Phys.Rev.Lett. 73 (1994), 573

[5] J.C. LaCombe et al., Phys.Rev.Lett. 83 (1999), 2997

[6] D.P. Corrigan et al., Phys. Rev. E 60 (1999), 7217

[7] C. Beckermann, Int. Mat. Rev. 47 (2002), 243

[8] W.D. Griffiths, D.G. McCartney, Mat.Sci.Eng. A 216 (1996), 47; P.A. Davidson, Ann.Review Fluid Mech. 31 (1999), 273

[9] R. H. Mathiesen et al., Met. Mat. Trans. A 37 (2006), 2515

[10] F. Kohler, Peritectic solidification of Cu-Sn alloys: microstructure competition at low speed, PhD Thesis 4037, EPFL, Switzerland, 2008

[11] M. Plapp, J. Cryst Growth 303 (2007), 49

V FIG. 3: Labyrinth-like lamellar microstructure observed in cross sections of a Cu- $21 \mathrm{wt} \% \mathrm{Sn}$ sample directionally solidified at $0.58 \mu \mathrm{m} / \mathrm{s}$ in a thermal gradient of $20 \mathrm{~K} / \mathrm{mm}$. Left panel: Continuous a-phase (in yellow-brown) with either lamellae or fibres of $\beta$ (in grey). Right panel: Isolated short lamellae and rods of $\alpha$ in a continuous $\beta$ phase [10].
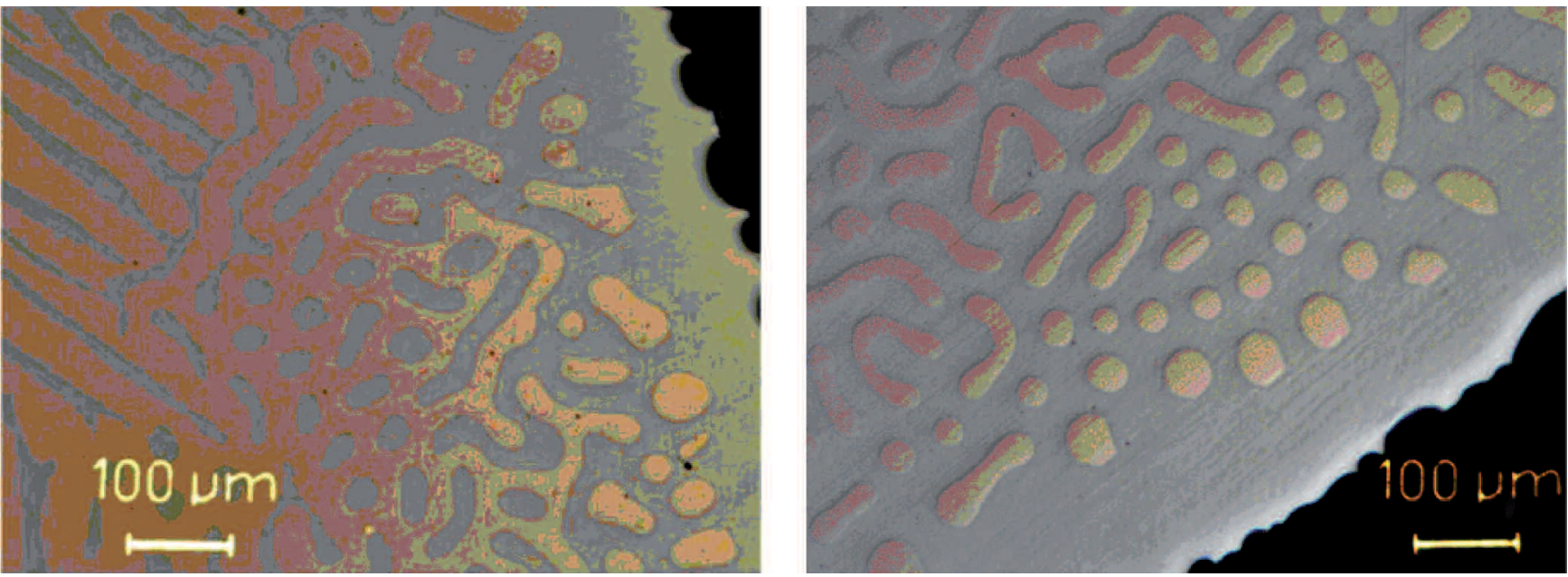